\title{
Phase Separation and Crystallization in Cu-Zr Metallic Glasses
}

\author{
N. Mattern ${ }^{1, *}$, U. Kühn ${ }^{1}$, A. Concustell ${ }^{2}$, A. Schöps ${ }^{3}$, M. D. Baro ${ }^{2}$ and J. Eckert ${ }^{1}$ \\ ${ }^{1}$ Leibniz-Institute for Solid State and Materials Research IFW Dresden, P.O. Box 2701 16, D-01171 Dresden, Germany \\ ${ }^{2}$ Department of Physics, University Barcelona, 08193 Bellaterra, Spain \\ ${ }^{3}$ HASYLAB at DESY, Notkestr. 85, D-22603 Hamburg, Germany
}

The structural behavior of rapidly quenched $\mathrm{Cu}-\mathrm{Zr}$ amorphous alloys was analyzed. High energy $\mathrm{X}$-ray diffraction patterns and atomic pair correlation functions exhibit monotonic changes with composition. The experimental results can be well described by a solid solution-like replacement of $\mathrm{Cu}$ and $\mathrm{Zr}$ atoms in the whole composition range. No indications are observed that would support the existence of phase separation in the supercooled liquid state of the binary Cu-Zr alloys. For $\mathrm{Cu}_{60} \mathrm{Zr}_{30} \mathrm{Ti}_{10}$ and $\mathrm{Cu}_{60} \mathrm{Zr}_{20} \mathrm{Ti}_{20}$ bulk metallic glasses the formation of ultrafine nanostructures are proven upon heating. The transformation starts below the glass transition temperature. Phase separation in $\mathrm{Cu}-\mathrm{Zr}-\mathrm{Ti}$ bulk metallic glasses is related to primary crystallization. [doi:10.2320/matertrans.MJ200708]

(Received November 29, 2006; Accepted December 28, 2006; Published June 25, 2007)

Keywords: metallic glasses, $X$-ray diffraction, phase separation, short-range order

\section{Introduction}

Cu-based bulk metallic glasses (BMG's) with high strength and about $2 \%$ fracture strain were first developed by Inoue et al. $^{1)}$ in the $\mathrm{Cu}-\mathrm{Zr}$-Ti and the $\mathrm{Cu}-\mathrm{Hf}-\mathrm{Ti}$ systems. Quite recently, high plasticity in compression tests was reported for binary $\mathrm{Cu}-\mathrm{Zr}^{2)}$ and ternary $\mathrm{Cu}-\mathrm{Zr}-\mathrm{Al} \mathrm{BMG}$ 's. ${ }^{3)}$ Nano-scale heterogeneities are discussed to be responsible for the improved ductility hindering the shear band propagation and creating multiple shear bands. A number of explanations for extraordinary plasticity and work hardening in monolithic BMG's have been suggested, including high Poisson's ratio, ${ }^{4}$ separation into two amorphous phases in the supercooled liquid, ${ }^{5)}$ formation of a nano-structured composite during casting, ${ }^{6}$ ) and nano-crystallization during deformation produced by the flow within the shear bands. ${ }^{2)}$ However, the mechanisms of structure formation and the transformation at the microscopic scale are not well understood so far. Earlier publications ${ }^{7,8)}$ reported the occurrence of phase separation in the undercooled liquid state of binary $\mathrm{Cu}-\mathrm{Zr}$ alloys.

The aim of this paper is to study the structural behavior of $\mathrm{Cu}$-based metallic glasses in a wide composition range. The analysis of the concentration dependence of structural and thermodynamic properties should give evidence whether or not phase separation in the $\mathrm{Cu}-\mathrm{Zr}$ glasses exists, which is of importance for understanding the mechanical properties of BMG's.

\section{Experimental}

Pre-alloyed ingots were prepared by arc-melting of elemental $\mathrm{Cu}, \mathrm{Zr}$ and $\mathrm{Ti}$ with a purity of $99.9 \%$ or better in a Ti-gettered argon atmosphere. For reaching homogeneity, the samples were remelted several times. From the $\mathrm{Cu}-\mathrm{Zr}$ pre-alloys, $3 \mathrm{~mm}$ wide and $25 \mu \mathrm{m}$ thick amorphous ribbons were prepared by means of rapid quenching from the melt using a single-roller melt-spinner under argon atmosphere.
Glassy samples of $\mathrm{Cu}_{60} \mathrm{Zr}_{30} \mathrm{Ti}_{10}$ and $\mathrm{Cu}_{60} \mathrm{Zr}_{20} \mathrm{Ti}_{20}$ with $3 \mathrm{~mm}$ diameter and $50 \mathrm{~mm}$ length were prepared by copper mold casting of the prealloys. Discs of $1 \mathrm{~mm}$ in height were cut from the rods. To analyze the short- and medium-range order, high energy X-ray diffraction experiments were conducted at the synchrotron beam-line BW5 (HASYLAB, Hamburg) using a wavelength of $\lambda=0.01250 \mathrm{~nm}$. Differential scanning calorimetry (DSC) experiments were performed employing a Netzsch DSC 404 calorimeter using a heating rate of $40 \mathrm{~K} /$ $\min$.

\section{Results and Discussion}

\subsection{Short-range order of amorphous $\mathrm{Cu}-\mathrm{Zr}$ versus composition}

Figure 1 shows experimental atomic pair correlation functions $g(r)$ of $\mathrm{Cu}-\mathrm{Zr}$ glasses calculated from the measured X-ray structure factors $S(q)$ by the Fourier transform: ${ }^{9)}$

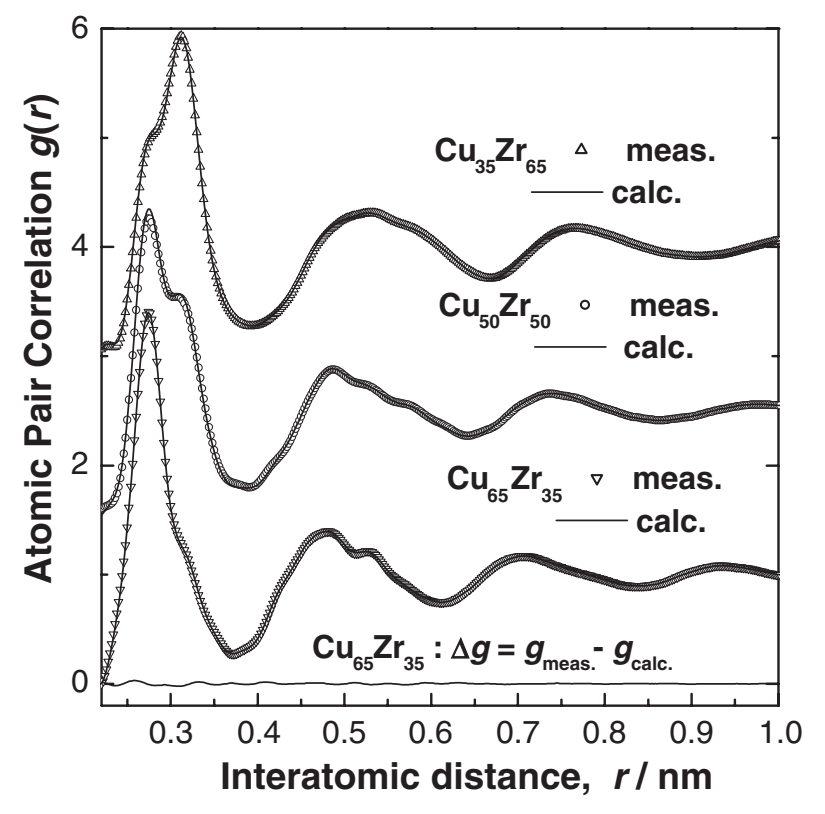

Fig. 1 Atomic pair correlation functions $g(r)$ of amorphous $\mathrm{Cu}_{x} \mathrm{Zr}_{100-x}$. 


$$
\begin{aligned}
4 \pi & \cdot r \cdot \rho_{0} \cdot(g(r)-1) \\
& =\frac{2}{\pi} \cdot \int(S(q)-1) \cdot q \cdot \sin (q \cdot r) \cdot d q
\end{aligned}
$$

where $\rho(r)$ is the atomic pair density distribution function and $\rho_{0}$ is the mean atomic density.

The $g(r)$ curves exhibit systematic changes for the different compositions. The total atomic pair correlation function $g(r)$ represents the weighted sum of the partial atomic pair correlation functions $g_{\mathrm{ij}}(r):^{9)}$

$$
g(r)=\sum_{i} \sum_{j} w_{i j} \cdot g_{i j}(r)
$$

The weight factors $w_{i j}=c_{i} \cdot c_{j} \cdot f_{i} \cdot f_{j} /\left(\sum_{i} c_{i} \cdot f_{i}\right)^{2}$ depend on the concentration $c_{i}$, and the atomic form amplitude $f_{i}$ is of the $i$-atom. For all $g(r)$ curves, two components of the first maximum are visible altering the heights with composition. The differences in $g(r)$ of the alloys are mainly related to the composition dependence of the weight factors $w_{\mathrm{ij}}$. The interatomic distance $r_{\mathrm{Zr}-\mathrm{M}}^{1}=0.275 \mathrm{~nm}$ corresponds to zirconium-metal distances in $\mathrm{Cu}-\mathrm{Zr}$ compounds, and $r_{\mathrm{Zr}-\mathrm{Zr}}^{1}=$ $0.312 \mathrm{~nm}$ to the zirconium-zirconium respectively. The $\mathrm{Cu}-$ $\mathrm{Cu}$ contributions are not resolved in the total $g(r)$ curve. It is not possible to determine the exact partial interatomic distances $r_{i j}$ and coordination number $N_{i j}$ from the total atomic pair correlation function $g(r)$ because the pair-wise contributions are superposed. But the 3 partial pair correlation $g_{i j}(\mathrm{r})$ functions can be extracted from the 3 experimental functions $g(r)$ by solving the linear equation system $g_{x}(r)=$ $\sum_{i} \sum_{j} w_{i j, x} \cdot g_{i j}(r)$. The applied procedure is based on the assumption that the partial functions $g_{\mathrm{ij}}(\mathrm{r})$ do not change with composition, which is similar to an isomorphous substitution. The correctness of that can be proven by the recalculation of the total atomic pair correlation functions from the 3 partial functions. Figure 1 compares the recalculations (lines) with the experimental curves (points). The difference curve $\Delta g=$ $g_{\text {mea. }}-g_{\text {calc. }}$ is also shown for amorphous $\mathrm{Cu}_{65} \mathrm{Zr}_{35}$. The good agreement results in a low mean square root values $\chi$ of $0.3 \%$ between measured and calculated $\mathrm{g}(\mathrm{r})$ curves, and demonstrates that the partial $g_{\mathrm{ij}}$ are not or only very weakly dependent on the chemical composition between 35 and 65 at\% $\mathrm{Cu}$.

Figure 2 shows the obtained 3 partial atomic pair correlation functions $g_{\mathrm{CuCu}}, g_{\mathrm{CuZr}}$, and $g_{\mathrm{ZrZr}}$. The position of the first maximum or the nearest neighbor distances $r_{\mathrm{Cu}-\mathrm{Cu}}=$ $0.248 \mathrm{~nm}, r_{\mathrm{Cu}-\mathrm{Zr}}=0.272 \mathrm{~nm}$, and $r_{\mathrm{Zr}-\mathrm{Zr}}=0.312 \mathrm{~nm}$ are in a general agreement with the data given in. ${ }^{10,11)}$ The $\mathrm{Cu}-\mathrm{Cu}$ distance $r=0.248 \mathrm{~nm}$ corresponds to the atomic diameter. The direct $\mathrm{Cu}-\mathrm{Cu}$ contact present in amorphous $\mathrm{Cu}-\mathrm{Zr}$ alloys deviates from the $\mathrm{Cu}$ environment of some of the crystalline counterparts pointing to differences in short-range order, e.g. in crystalline $\mathrm{CuZr}_{2}$ the $\mathrm{Cu}$ atoms are not nearest neighbors at all, and in the bcc $\mathrm{CuZr}$ phase, the smallest $\mathrm{Cu}-\mathrm{Cu}$ distance is equal to the $\mathrm{Cu}-\mathrm{Zr}$ distance. ${ }^{12)}$ From the partial pair correlation function $g_{\mathrm{ij}}$ the partial nearest neighbor numbers $N_{\mathrm{ij}}$ can be calculated by the integration over of the first maximum. Figure 3 shows the composition dependence of the partial nearest neighbor numbers. The calculated values are proportional to the corresponding element concentrations. The assumption of unchanged partial pair correlation

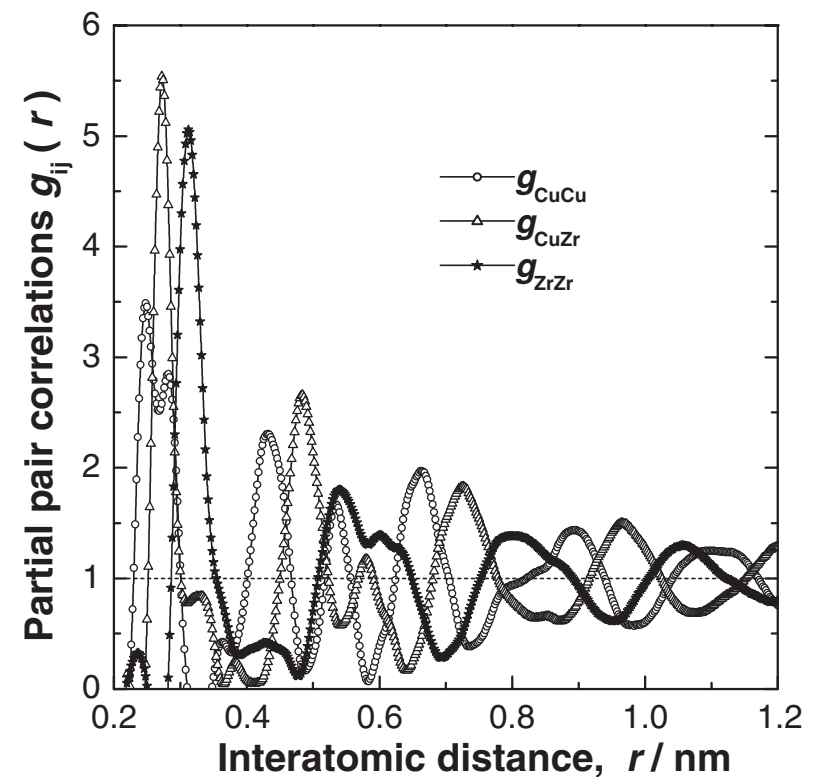

Fig. 2 Partial atomic pair correlation functions $g_{i j}(r)$ of amorphous $\mathrm{Cu}_{x} \mathrm{Zr}_{100-x}$

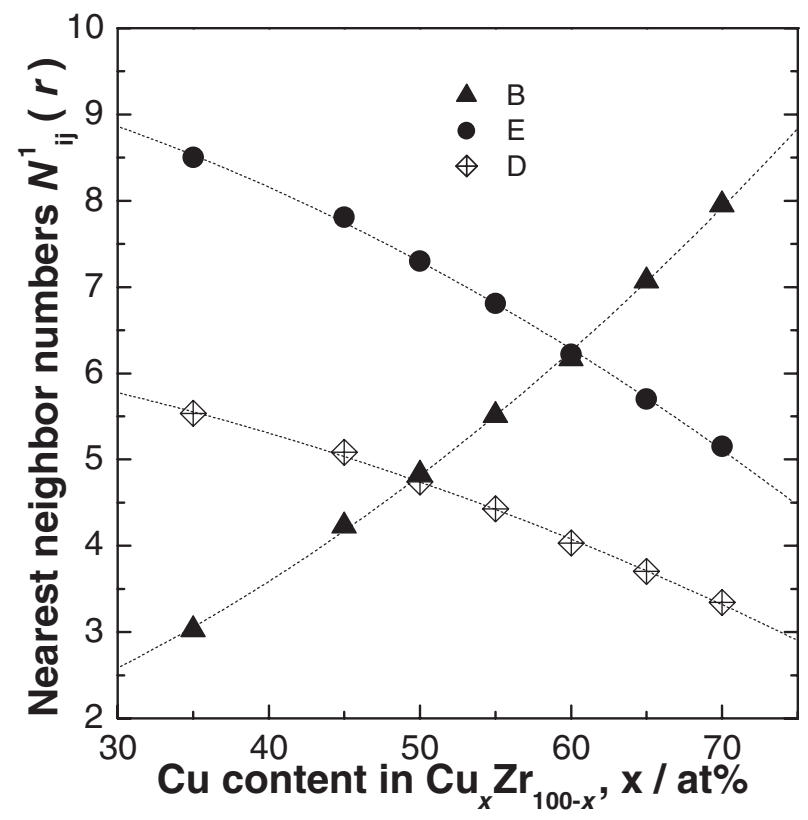

Fig. 3 Partial nearest neigbor numbers $N^{1}$ ij of amorphous $\mathrm{Cu}_{x} \mathrm{Zr}_{100-x}$. (7B: $\mathrm{Cu}-\mathrm{Cu}, \mathrm{E}: \mathrm{Zr}-\mathrm{Zr}$, D0: $\mathrm{Cu}-\mathrm{Zr}$ )

functions implies directly a continuous solid solution behavior with composition. The experimental results are well described by a solid solution-like replacement of $\mathrm{Cu}$ and $\mathrm{Zr}$ atoms in the composition range between 30 and $70 \mathrm{at} \% \mathrm{Cu}$. Therefore, no indication is observed for the existence of phase separation in the supercooled liquid state of the binary $\mathrm{Cu}-\mathrm{Zr}$ system.

\subsection{Nano-phase formation in $\mathrm{Cu}_{60} \mathrm{Zr}_{40-x} \mathrm{Ti}_{x}$ bulk me- tallic glass}

Figure 4 compares the crystallization behavior of the amorphous $\mathrm{Zr}_{60} \mathrm{Zr}_{40-x} \mathrm{Ti}_{x}$ alloys by their DSC curves. The crystallization temperature of amorphous $\mathrm{Cu}_{60} \mathrm{Zr}_{40}$ decreases 


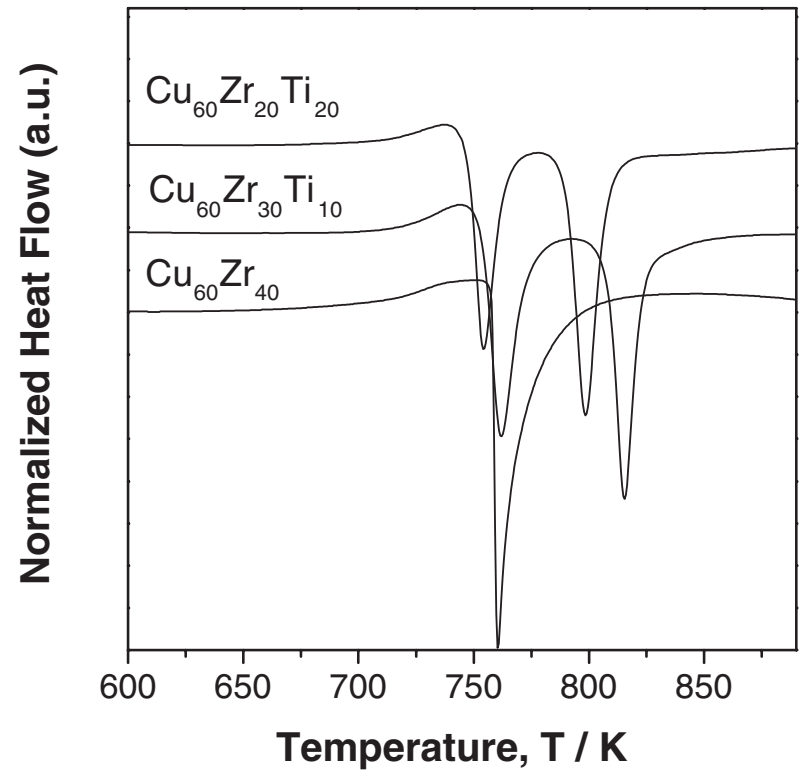

Fig. 4 DSC scan of amorphous $\mathrm{Cu}_{60} \mathrm{Zr}_{40-x} \mathrm{Ti}_{x}(40 \mathrm{~K} / \mathrm{min})$.

Table 1 Glass transition and crystallization temperatures (onset), mass and atomic density of amorphous $\mathrm{Cu}_{60} \mathrm{Zr}_{40-x} \mathrm{Ti}_{x}$ alloys.

\begin{tabular}{lcccc}
\hline \multicolumn{1}{c}{ Alloy } & $T_{g}(\mathrm{~K})$ & $T_{x}(\mathrm{~K})$ & $\sigma \mathrm{gcm}^{-3}$ & $\rho_{0} \mathrm{~nm}^{-3}$ \\
\hline $\mathrm{Cu}_{60} \mathrm{Zr}_{40}$ & 719 & 755 & 7.53 & 60.8 \\
$\mathrm{Cu}_{60} \mathrm{Zr}_{30} \mathrm{Ti}_{10}$ & 714 & 750 & 7.41 & 61.6 \\
$\mathrm{Cu}_{60} \mathrm{Zr}_{20} \mathrm{Ti}_{20}$ & 712 & 745 & 7.24 & 63.5 \\
\hline
\end{tabular}

with Ti content and changes from a single step crystallization into two or three steps for the Ti containing alloys. The characteristic values of the glass transition temperatures $T_{g}$ and crystallization temperatures $T_{x}$ are summarized in Table 1 . The values are in a good agreement with data given in Ref. 1), 13). In the case of $\mathrm{Cu}_{60} \mathrm{Zr}_{40}$ the orthorhombic $\mathrm{Cu}_{10} \mathrm{Zr}_{7}$ phase is formed with a size of the crystals of about $100 \mathrm{~nm}$. For $\mathrm{Cu}-\mathrm{Zr}-\mathrm{Ti}$ alloys recent studies reported that these BMGs are composites of nanocrystals dispersed in metallic glass matrix. ${ }^{13-15)} \mathrm{X}$-ray diffraction was discussed to show no evidence for the presence of crystalline phase. ${ }^{15)}$ On the other hand, Nagahama et al. ${ }^{16)}$ recently could convincingly show that depending on the cooling rate fully amorphous or partly crystallized $\mathrm{Cu}_{60} \mathrm{Zr}_{30} \mathrm{Ti}_{10}$ are prepared.

To analyze the structural transition in detail, samples were heated in the DSC to temperatures before and after the first DSC event followed by rapidly cooling down to room temperature $(100 \mathrm{~K} / \mathrm{min})$. Figure 5 shows the structure factor $S(q)$ of annealed amorphous $\mathrm{Cu}_{60} \mathrm{Zr}_{30} \mathrm{Ti}_{10}$ and $\mathrm{Cu}_{60} \mathrm{Zr}_{20} \mathrm{Ti}_{20}$ samples. The structure factors of samples annealed up to temperatures before the first DSC event are very similar to those of the as-cast material. After heating up above the first exothermic peak small differences appear in $S(q)$. The heights of the first, second and further maxima of the structure factor increase but the diffuse character of the diffraction patterns remains. The difference curves between the structure factors of the samples heated to temperatures before $\left(T_{1}\right)$ and after $\left(T_{2}\right)$ of the first DSC event $\Delta S(q)=$

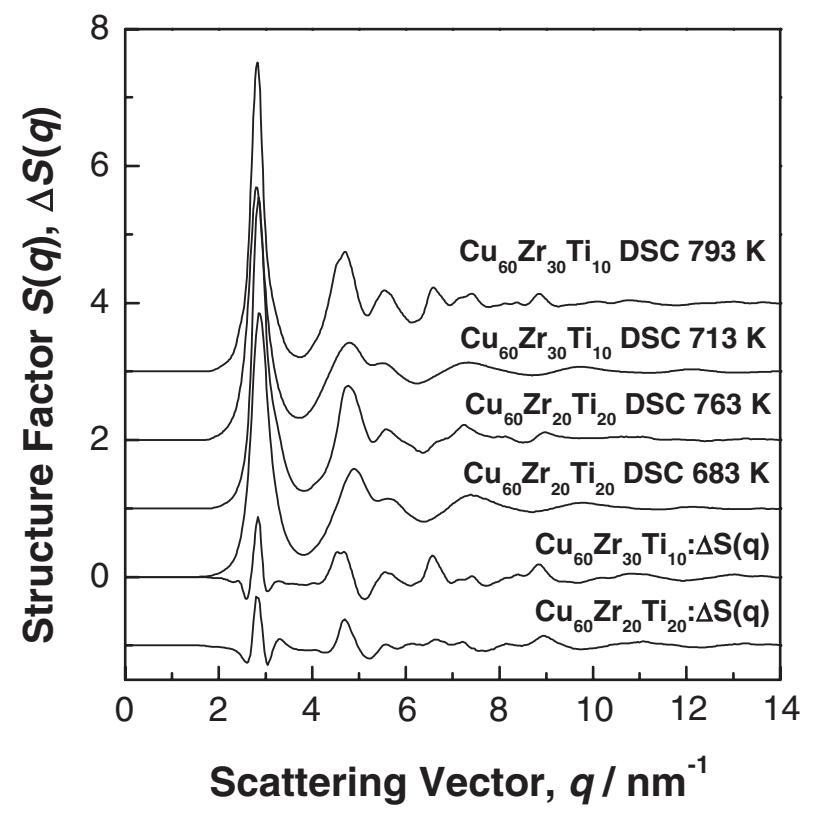

Fig. 5 Structure factor $S(q)$ of amorphous $\mathrm{Cu}_{60} \mathrm{Zr}_{40-x} \mathrm{Ti}_{x}$ annealed before and after first DSC event, and difference curves $\Delta S(q)$.

$S_{T 2}(q)-S_{T 1}(q)$ are also given in Fig. 5. The difference curves exhibit clearly changes in $S(q)$ especially for the maxima at higher $q$-values not detectable in standard XRD measurements. ${ }^{15}$

The microstructure of the specimens was analyzed by high-resolution transmission electron microscopy (HREM). Figure 6 shows TEM image of the $\mathrm{Cu}_{60} \mathrm{Zr}_{20} \mathrm{Ti}_{20}$ annealed near $T_{g}$ at $T=703 \mathrm{~K}$ for $25 \mathrm{~min}$. An inhomogeneous nanostructure is visible indicating the formation of nanocrystals of around $5 \mathrm{~nm}$ in size. The image contrast in Fig. 6 indicates that there could be chemical differences between the crystals and the matrix present. X-ray spectroscopy point analysis in scanning transmission operating mode (STEM) with a $1 \mathrm{~nm}$ nanobeam gives an increase of the $\mathrm{Cu}$ signal, a constant $\mathrm{Zr}$ signal and a reduced $\mathrm{Ti}$ signal for the nanocrystals in comparison with the surrounding matrix. These findings are in a general agreement with the results of Nagahama et al. ${ }^{16)}$

The atomic pair correlation functions $g(r)=\rho(r) / \rho_{0}$ were calculated to analyse the structural changes in real space. Figure 7 shows the calculated $g(r)$ curves for $\mathrm{Cu}_{60} \mathrm{Zr}_{30} \mathrm{Ti}_{10}$ samples annealed at temperatures before and after the first DSC event. Clearly differences in short- and medium-range order are visible. The inter-atomic distances of the first neighborhood are altered in the transformed phase. The correlation length expressed by the extension of the oscillation in $\mathrm{g}(\mathrm{r})$ becomes larger but does not exceed $3 \mathrm{~nm}$ in accordance with the TEM results. The experimental pair correlation functions can be compared with those of known crystalline phases in the $\mathrm{Cu}-\mathrm{Zr}$-Ti system. Neither bcc $\mathrm{Cu}-\mathrm{Zr}$, fcc $\mathrm{Cu}, \mathrm{Cu}_{10} \mathrm{Z}_{7}$ nor $\mathrm{Cu}_{51} \mathrm{Zr}_{14}$ are able to describe the shortrange order of the experimental curve. A rather good agreement is found with the atomic pair correlation function of the cubic Laves phase $\mathrm{Cu}_{5} \mathrm{Zr}$ shown in Fig. 7 which is also consistent with the results of elemental analysis in the TEM. Similar agreement is obtained for the $\mathrm{Cu}_{60} \mathrm{Zr}_{20} \mathrm{Ti}_{20}$ sample 


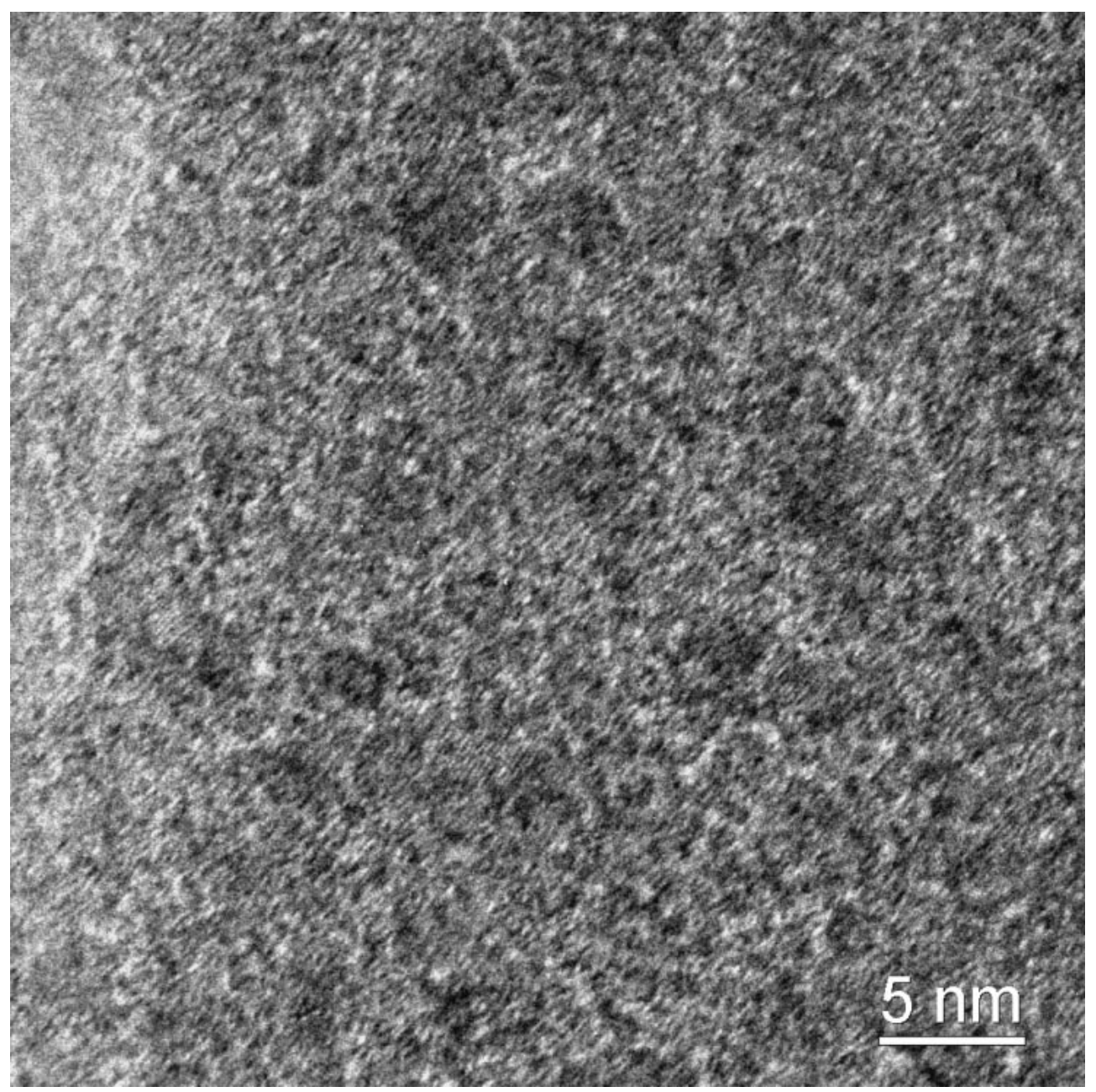

Fig. 6 TEM image of $\mathrm{Zr}_{60} \mathrm{Cu}_{20} \mathrm{Ti}_{20}$ annealed at $703 \mathrm{~K}$ for $25 \mathrm{~min}$.

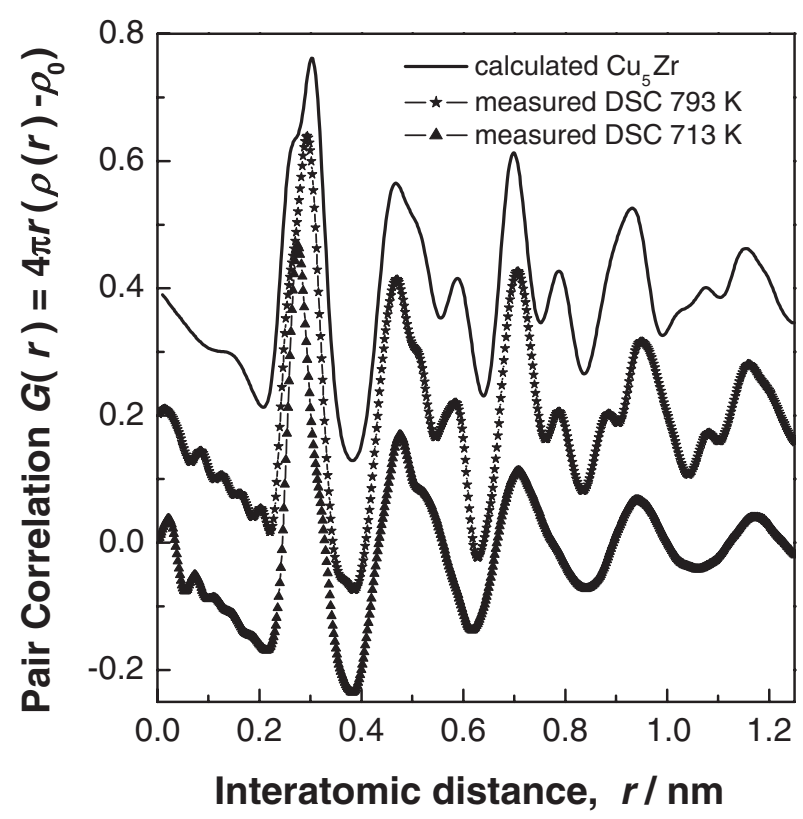

Fig. 7 Reduced atomic pair correlation functions $G(r)$ of $\mathrm{Cu}_{60} \mathrm{Zr}_{30} \mathrm{Ti}_{10}$.

annealed above the first DSC peak. The comparison point to the formation of an intermetallic $\mathrm{Cu}-\mathrm{Zr}$ nanophase as the first step of crystallization.

\section{Conclusions}

There is no indication of phase separation into two amorphous phases in binary $\mathrm{Cu}-\mathrm{Zr}$ and ternary $\mathrm{Cu}-\mathrm{Zr}-\mathrm{Ti}$ bulk metallic glasses. Monotonic changes of structural parameters in amorphous $\mathrm{Cu}-\mathrm{Zr}$ with composition point to a solid solution-like replacement of $\mathrm{Cu}$ and $\mathrm{Zr}$ atoms. Phase separation in $\mathrm{Cu}-\mathrm{Zr}$-Ti bulk metallic glasses is related to the formation of an ultrafine nanostructure by primary crystallization.

\section{Acknowledgement}

HASYLAB Hamburg is gratefully acknowledged for the support during synchrotron beam time. The authors thank B. Arnold, H. Frey, S. Kuscinsky, D. Lohse, B. Opitz, and A. Oswald for technical assistance.

\section{REFERENCES}

1) A. Inoue, W. Zhang, T. Zhang and K. Kurosaka: Acta Mater. 49 (2001) 2645-2652.

2) M. Chen, A. Inoue, W. Zhang and T. Sakurai: Phys. Rev. Lett. 96 (2006) 245502-1-245502-4.

3) J. Das, M. B. Tang, K. B. Kim, R. Theismann, F. Baier, W.H. Wang and J. Eckert: Phys. Rev. Lett. 94 (2005) 205501-205504.

4) J. Schroers and W. L. Johnson: Phys. Rev. Lett. 93 (2004) 255506. 
5) K. B. Kim, J. Das, F. Baier, M. B. Tang, W. H. Wang and J. Eckert: Appl. Phys. Lett. 88 (2006) 051911,1-3.

6) J. Saida, T. Osuna, A. Inoue and M. Onuma: J. Mat. Res. 18 (2003) 2013-2016.

7) T. W. Barbee, R. G. Walmsley, A. F. Marshall, D. L. Keith and D. A. Stevenson: Appl. Phys. Lett. 38 (1981) 132-135.

8) R. Bormann: Habilitation Thesis,University Göttingen (1988).

9) C. N. J. Wagner: Liquid Metals, Chemistry and Physics, ed. by S. Z. Beer (Marcel Dekker Inc., New York, 1972), p. 257.

10) H. S. Chen and Y. Waseda: Phys. Stat. Sol. (a) 51 (1979) 593.

11) M. Laridjani and J. F. Sadoc: J Non-Cryst. Solids, 106 (1988) 42.
12) P. Villars and L. D. Calvert: Pearson's Handbook of Crystallographic Data for Intermetallic Alloys (ASM International, Ohio, 1985).

13) N. Kasai, J. Saida, M. Matsushita, T. Osuna, E. Matsubara and A. Inoue: J. Phys. Condens Matter 14 (2002) 13867-13877.

14) Y. Chen, T. Zhang, W. Zhang, D. H. Ping, K. Hono, A. Inoue and T. Sakurai: Mater. Trans. 43 (2002) 2647.

15) J. Z. Jiang, J. Saida, H. Kato, T. Ohsuna and A. Inoue: Appl. Phys. Lett. 82 (2003) 4041-4043.

16) D. Nagahama, T. Ohkubo, T. Mukai and K. Hono: Mater. Trans. 46 (2005) 1264-1270. 